\title{
Wavelength Domain Multiplexed fiber specklegram sensor
}

\author{
L. Rodriguez-Cobo*, M. Lomer, A. Cobo, E. Real and J.M. Lopez-Higuera \\ Photonics Engineering Group, University of Cantabria, 39005, Santander (Spain)
}

\begin{abstract}
In this work, a low-cost WDM interrogation system has been proposed and experimentally tested to obtain two independent channels of a fiber specklegram sensor. Two lasers of different wavelengths have been launched into two multimode Polymer Optical Fibers (POFs) and then combined through a coupler before their interrogation using a RBG camera. By analyzing each color of the video sequence, the two fiber channels can be independently obtained. Besides, the speckle sensitivity has been also studied by analyzing different properties of speckle patterns such as their contrast or the speckle size. The achieved results help to the development of new fiber specklegram sensors by allowing a direct comparison between two specklegrams of different properties.
\end{abstract}

Keywords: Fiber Specklegram Sensor, Polymer Optical Fiber, WDM

\section{INTRODUCTION}

Fiber Specklegram Sensors (FSSs) have been widely developed during last years [1], where high sensitivities can be achieved employing low cost technologies, and particularly, CCD cameras. The charge-coupled device (CCD), invented by Boyle and Smith in 1969 [2] works thanks to the photoelectric effect, and plays an important role in scientific researches in different fields. They became relevant when, in the 1980s, these devices replace conventional photographs in the field of astronomy [3]. Within FSSs, the CCD is placed on the output end of the fiber to record the images with the distribution of the optical field. When a coherent light propagates within a multimode fiber, an intermodal interference takes place along the fiber path, provoking speckle patterns at the fiber exit. Each speckle intensity varies with any change on the propagation medium (multimode fiber), so, by analyzing speckle pattern variations, these changes can be measured. A CCD camera can be employed to capture these changes and, with a suitable processing scheme, a signal proportional to the perturbations can be obtained. Different advances during last years have benefited the CCD technologies, achieving higher resolutions, but reducing their size and cost. These perspectives allow their application in many sensing scenarios, as shown recently Richards et al., in the study of blood flow using the webcam camera [4].

In this work, a proof-of-concept multipoint fiber specklegram sensor has been presented by coding two independent channels using different wavelengths. Both signals have been coupled to a single multimode fiber using a 50-50 coupler and connected to a commercial color camera. Different properties of the FSS have been analyzed in terms of the final sensor sensitivity.

\section{SPECKLE SENSITIVITY}

The sensitivity of a Fiber Specklegram Sensor is given by the amount and contrast of speckles comprised within the captured images. These values are mainly affected by three elements: the light source, the type of multimode fiber and the detection device [5-6]. The contrast of speckles is related to the coherence length of the source $\left(L_{c}\right)$ and has influence on the final sensitivity, thus, employing a more coherent light source, a greater sensitivity can be achieved.

The amount of speckles within a specific detecting area (e.g. CCD area) is given by two features, the number of modes and the averaged size of speckles. For a step-index fiber, the number of modes $(M)$ is given by $M=V^{2} / 2$, being $V=$ $(2 \pi a / \lambda)(N A)$, where $N A$ is the numerical aperture, $a$ is the core radius and $\lambda$ the laser wavelength. When the speckle pattern is projected, the average size of speckle $(T)$, is given by $T \approx d(\lambda / a)$, where $d$ is the distance between the fiber and the detector's surface. Based on these relations, as the wavelength increases, the number of modes becomes lower but the speckle size increases, reducing the final sensitivity.

*luis.rodriguez@unican.es; phone +34 942200877; fax +34 942200877

23rd International Conference on Optical Fibre Sensors, edited by José Miguel López-Higuera,

Julian Jones, Manuel López-Amo, José Luis Santos, Proc. of SPIE Vol. 9157, 91579K

(C) 2014 SPIE · CCC code: $0277-786 X / 14 / \$ 18 \cdot$ doi: $10.1117 / 12.2059553$ 


\section{EXPERIMENTS}

Two fiber specklegram sensors have been combined into a single multimode fiber, taking advantage of the three color channels offered by RGB cameras. Each specklegram channel has been obtained with a different laser: a cheap laser diode centered on $532 \mathrm{~nm}$ and a He-Ne laser emitting at $632.8 \mathrm{~nm}$. Those wavelengths have been chosen to match two filters of the RGB camera, trying to avoid cross-talk between channels. The setup employed for the experimental characterization is depicted in Fig. 1.

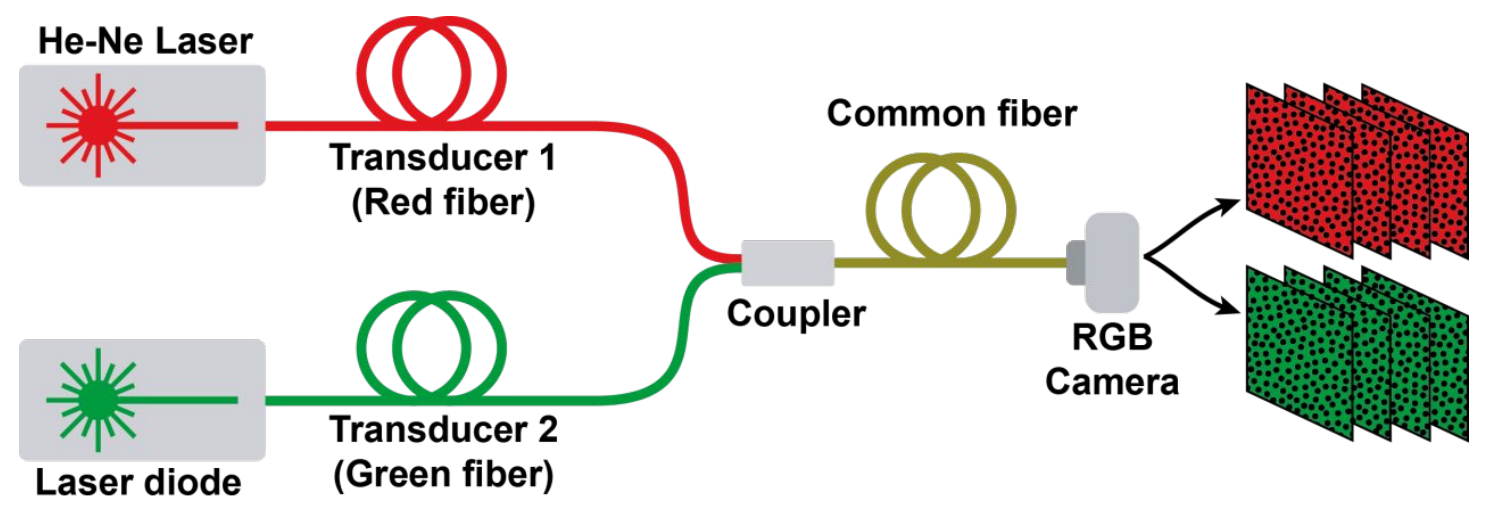

Figure 1. Experimental setup. Two lasers of different wavelengths are launched into different POFs. The same RGB camera is employed to the interrogation.

Both laser signals were launched into $1 \mathrm{~m}$ of multimode POFs of $980 \mathrm{um}$ and then combined using a 50-50 coupler. The output of the coupler was directly connected to a CCD camera. The power difference between channels has been compensated by scaling each of the employed channels (Red and Green) by its mean value. This process can be noticed in Fig. 2.
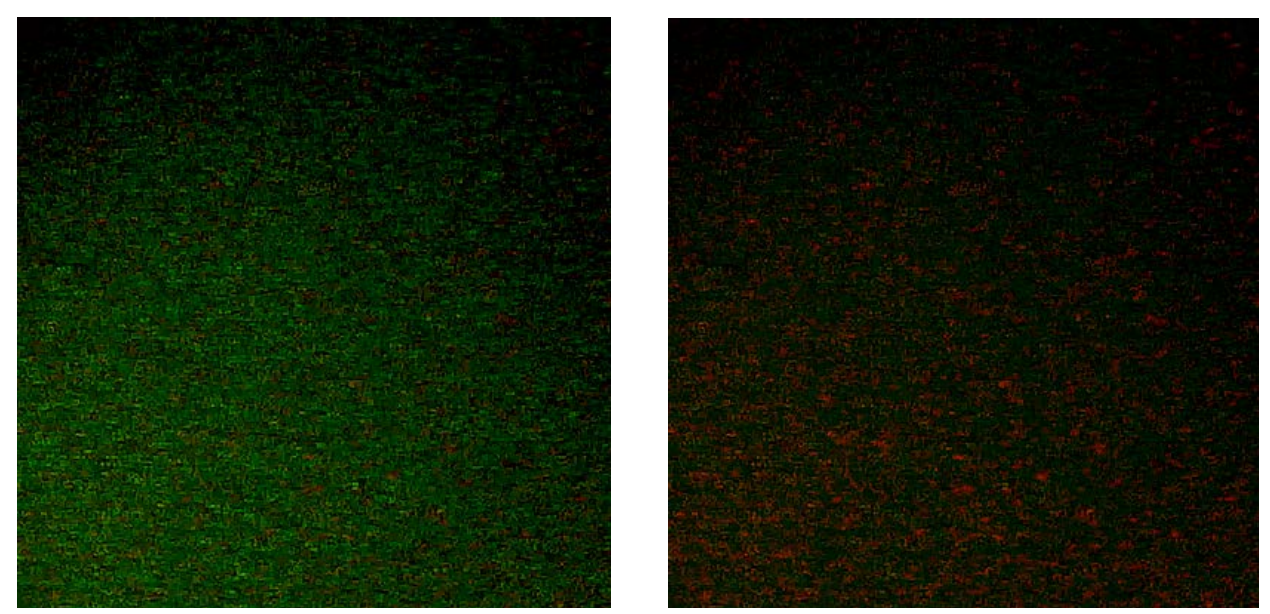

Figure 2. Captured specklegram of 400 by 400 pixels (left). The same specklegram has been equalized to compensate the power difference between channels (right).

The employed lasers are based on very different technologies what has influence to the final sensitivity of the specklegram sensors. Particularly, the lasers have very different coherent lengths (several meters vs. less than one mm) that is directly related to the contrast and modifies the sensitivity of the specklegram sensor. On the other hand, laser wavelengths are different, what produces different speckle sizes, having also influence on the final sensitivity. 


\section{CONTRAST AND DIFFERENTIAL PROCESSING SCHEME}

Besides the wavelength, the visibility of speckles have a great influence on the final sensitivity of the sensor. Trying to experimentally verify this influence, the contrast of both channels has been obtained. Since the areas of lower intensity of speckle patterns are not detected with the employed setup, the RMS contrast [7] has been employed to determine the quality of the chosen lasers. This metric can be defined as:

$$
\mathrm{RMS}=\sqrt{\frac{1}{M N} \sum_{n=0}^{N-1} \sum_{m=0}^{M-1}\left(I_{n m}-\bar{I}\right)^{2}}
$$

where intensities $I_{n m}$ are the n-th m-th element of the two dimensional specklegram of size M by N. $\bar{I}$ is the average intensity of all pixel values of the specklegram. The two speckle patterns obtained from the same capture have .
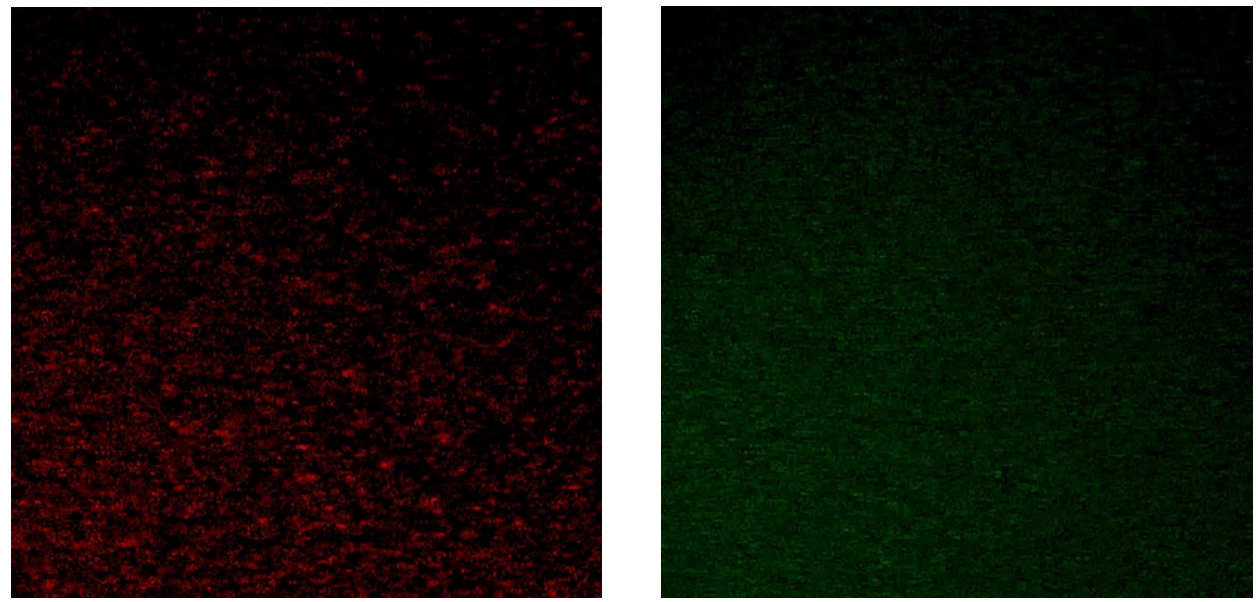

Figure 3. Red (left) and green (right) channels of the equalized specklegram. Red specklegram has a better contrast $(0.06)$ than the green (0.03), improving the visibility of the speckles.

A simple differential processing scheme has been employed to verify the sensitivity dependence with the speckle contrast and wavelength. A differentially processed sequence for two specklegrams of NxM pixels can be defined as follows (Eq. 2):

$$
\mathrm{D}\{\mathrm{i}\}=\frac{1}{K \cdot M N} \sum_{n=0}^{N-1} \sum_{m=0}^{M-1}\left|I_{n m}^{i-1}-I_{n m}^{i}\right|
$$

where $\mathrm{K}$ is the full scale value of the specklegram color map (e.g. $\mathrm{K}=255$ for 8-bit grayscale) and $I_{n m}^{i}$ corresponds to the pixel of the n,m position of the i-th specklegram. The differential intensity variation is proportional to the small variations caused by the perturbation between two consecutive specklegrams, so it can very useful to experimentally analyze the sensitivity to external perturbations.

\section{RESULTS}

Based on the setup detailed in Fig. 1, different mechanical perturbations have been applied to both fibers. Several initial test have performed to determine the influence of the cross-talk between different channels of the RBG camera. By switching off one laser, the signal was analyzed in both channels, with and without perturbation on the individual fibers. The achieved results proved the absence of cross-talk, on the employed setup, simplifying the processing scheme. The same perturbations have been applied to the individual fibers and to the common one. Some of the achieved results are depicted below. 

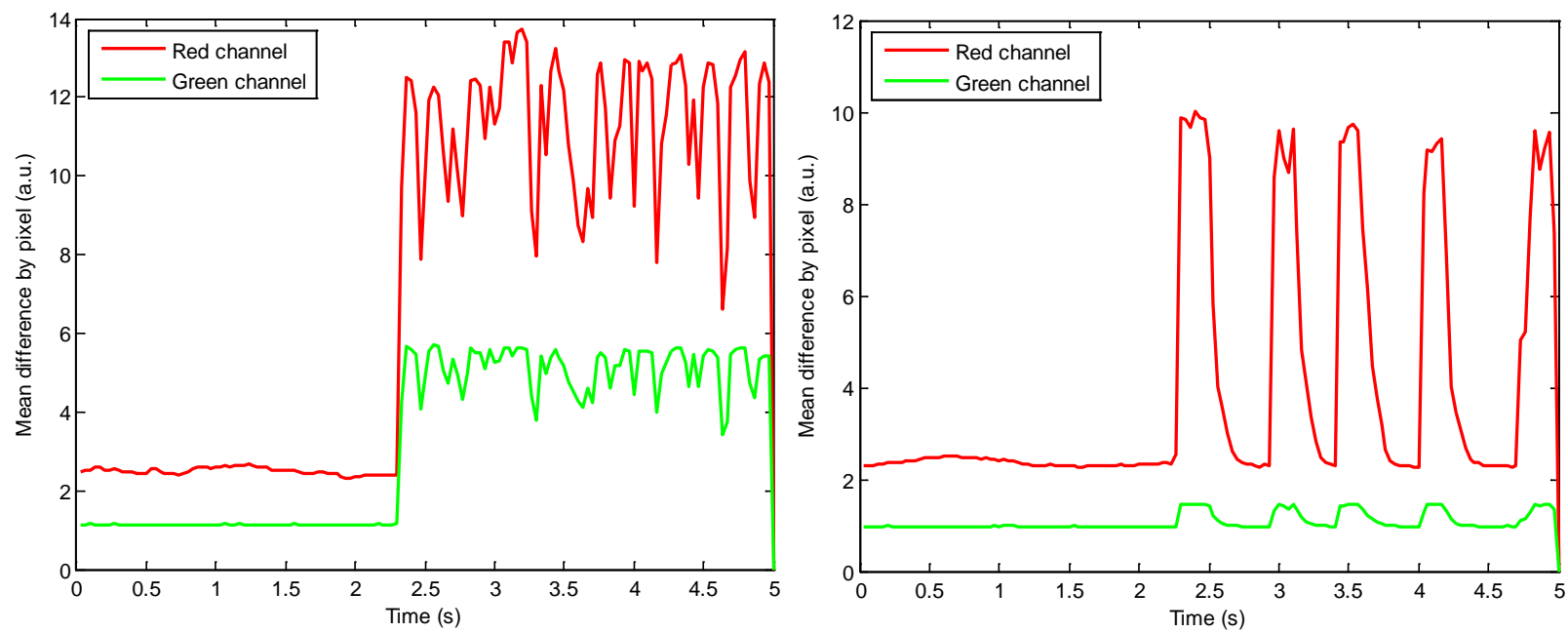

Figure 4. Differential processed sequences of red and green channels under a perturbation on the common fiber (left) and on the red fiber (right).

In Fig 4 (left), the differential processed sequence of each channel is depicted when an external perturbation was applied to the common fiber. After equalizing the power between channels, the red channel exhibit a greater sensitivity than the green, as the contrast suggests. In Fig 4 (right), the perturbation was just applied to the red fiber, however, some slight perturbation was superficially transmitted to the green fiber through the fixing table and it can be noticed, proving the extreme sensitivity of fiber specklegram sensors.

\section{CONCLUSIONS}

In this work, a new wavelength domain multiplexed specklegram sensor has been experimentally demonstrated. Employing two lasers centered on the filters of a RGB camera, two individual speckle channels can be retrieved employing a low cost setup. Several aspects of employed lasers which contribute to the final sensitivity of specklegram sensors have been also studied, proving the importance of the coherent length ( related to the contrast) over the laser wavelength (related to the speckle size). The achieved results show the possibilities of specklegram sensors to obtain independent measurements using low cost equipments by coding each channel using different laser wavelengths. This technology can be employed to develop multipoint-multiparameter sensors based on commercial CCD color cameras. This work has been supported by the project TEC2010-20224-C02-02 and grant AP2009-1403.

\section{REFERENCES}

[1] K. Peters, "Polymer optical fiber sensors: A review," Smart materials and structures, 20, pp. 013002 (2010).

[2] W.S. Boyle and G.E. Smith, "Charge Coupled Semiconductor Devices," Bell Systems Technical Journal, 49, pp. 587-593 (1970).

[3] S.B. Howell, Handbook of CCD Astronomy, Cambridge University Press, Second edition, Cambridge, UK (2006).

[4] L.M. Richards, S.M.S. Kazmi, J.L. Davis, K.E. Olin, and A.K. Dunn, "Low-cost laser speckle contrast imaging of blood flow using a webcam”, Biomed. Opt. Express 4(10), 2269-2283 (2013).

[5] J.W. Goodman, "Some fundamentals properties of speckle”, J. Opt. Soc. Am., 66(11), 1145-1150 (1976).

[6] J.W. Goodman, Speckle Phenomena in Optics, Roberts and Company, Chapter 7, Englewood, Colorado, (2007).

[7] E. Peli, “Contrast in Complex Images”, J. Opt. Soc. Am. A, 7(10), 2032-2040 (1990). 